\title{
ON A CLASSIFICATION OF INTEGRAL FUNCTIONS BY MEANS OF CERTAIN INVARIANT POINT PROPERTIES*
}

BY

R. D. CARMICHAEL, W. T. MARTIN, $\dagger$ AND M. T. BIRD

1. Introduction. In the investigation of linear differential equations of infinite order and of certain other types of linear functional equations, the authors of this paper have severally had occasion to employ certain point properties of integral functions which remain unaltered in passing from one regular point to another. These invariant point properties are of considerable interest in themselves, and the theorems to which they give rise extend classic results in the theory of integral functions. In this paper we lay the foundations of a general theory of these invariant point properties of integral functions and present the principal results concerning them which we have severally drawn upon in our recent investigations (not yet published).

2. On $I$-sequences. Throughout the paper the symbol $\left\{t_{n}\right\}$ will denote an infinite sequence of positive numbers $t_{0}, t_{1}, t_{2}, \cdots$. We define associated positive-termed sequences $\left\{\lambda_{n}\right\}$ and $\left\{\rho_{n}\right\}$ by means of the relations

$$
t_{0}=\lambda_{0}=\rho_{0}, \quad t_{n}=\lambda_{1} \lambda_{2} \cdots \lambda_{n}=\left(\rho_{n}\right)^{n}, \quad(n=1,2,3, \cdots) .
$$

An infinite sequence $\left\{t_{n}\right\}$ will be called an $I$-sequence when and only when

(a) it consists of positive numbers having the property that for every single-valued analytic function $F(x)$ we have

$$
\limsup _{n=\infty}\left|t_{n} F^{(n)}\left(x_{0}\right) / n !\right|^{1 / n}=\limsup _{n=\infty}\left|t_{n} F^{(n)}\left(x_{1}\right) / n !\right|^{1 / n}
$$

for every two finite regular points $x_{0}$ and $x_{1}$ of $F(x)$;

(b) there exists at least one analytic function $F(x)$ for which the superior limits in (1) have a value different from zero.

The property of $F(x)$ expressed by (1) is an invariant point property of of the function.

If $\left\{t_{n}\right\}$ is an $I$-sequence and if $F(x)$ is a particular analytic function for which the superior limits in (1) have the value $q(0 \leqq q \leqq \infty)$ then we shall say that $F(x)$ is of type $\left(t_{0}, t_{1}, t_{2}, \cdots ; q\right)$, or, more briefly, of type $\left(t_{n} ; q\right)$. When $q$ is finite we shall speak of the type as normal.

* Presented to the Society, April 11, 1936; received by the editors February 21, 1936.

$\dagger$ National Research Fellow. 
It is obvious that every polynomial is of type $\left(t_{n} ; 0\right)$ for every $I$-sequence $\left\{t_{n}\right\}$. Hence any particular $I$-sequence serves to separate all analytic functions into (two or more) types or classes.

If $\left\{t_{n}\right\}$ is an $I$-sequence it is clear that $\left\{\tau_{n}\right\}$ is also an $I$-sequence provided that its elements are positive and that the limit

$$
\lim _{n=\infty}\left(\tau_{n} / t_{n}\right)^{1 / n}
$$

exists as a finite quantity $p$ different from zero. Then if $F(x)$ is of type $\left(t_{n} ; q\right)$ it is also obviously of type $\left\{\tau_{n} ; p q\right\}$.

Let us put

$$
\limsup _{n=\infty}\left(t_{n}\right)^{1 / n}=\rho, \quad \liminf _{n=\infty}\left(t_{n}\right)^{1 / n}=\sigma, \quad(0 \leqq \sigma \leqq \rho \leqq \infty) .
$$

We shall first prove that $\rho$ is necessarily infinite for every $I$-sequence $\left\{t_{n}\right\}$. For, if we assume $\rho$ to be finite, then for the particular function $F(x)=1 /(1-x)$ relation (1) leads to the equation

$$
\rho\left|1-x_{0}\right|^{-1}=\rho\left|1-x_{1}\right|^{-1}
$$

for all finite $x_{0}$ and $x_{1}$ different from unity; and this is impossible unless $\rho=0$. But if $\rho=0$ it is easy to see that condition (b) in the definition of an $I$-sequence is not satisfied.

When $\sigma \neq 0$ we denote by $E(x)$ the function

$$
E(x)=\sum_{\nu=0}^{\infty} \frac{x^{\nu}}{t_{\nu}}
$$

the indicated power series converging for $|x|<\sigma$. Taking $x_{0}=0$ and $x_{1}$ such that $\left|x_{1}\right|<\sigma$ we have from (1) the relation

$$
1=\limsup _{n=\infty}\left|t_{n} E^{(n)}\left(x_{1}\right) / n !\right|^{1 / n}
$$

provided that $\left\{t_{n}\right\}$ is an $I$-sequence, as we now assume. Hence $E(x)$ is analytic in the interior of a circle about $x_{1}$ as center and of radius $\sigma$. But this is not possible for every $x_{1}$ satisfying the indicated condition unless $\sigma$ is infinite, since if $\sigma$ is finite $E(x)$ has a singularity on the circle $|x|=\sigma$.

On combining the results obtained, we have the following theorem:

THEOREM I. In order that $\left\{t_{n}\right\}$ shall be an I-sequence, it is necessary that one or the other of the two following conditions shall be satisfied:

$$
\lim _{n=\infty}\left(t_{n}\right)^{1 / n}=\infty ;
$$

$$
\limsup _{n=\infty}\left(t_{n}\right)^{1 / n}=\infty \quad \text { and } \quad \lim _{n=\infty} \inf \left(t_{n}\right)^{1 / n}=0 .
$$


An $I$-sequence $\left\{t_{n}\right\}$ for which condition (4) [(5)] is satisfied will be called an $A$-sequence [an $\alpha$-sequence]. We shall be concerned with $A$-sequences. We have not been able to determine whether $\alpha$-sequences exist; we know some of their properties if they do exist; but we shall not present those properties in this paper.

3. On $A$-sequences. For every $A$-sequence $\left\{t_{n}\right\}$ the function $E(x)$ defined in (3) is an integral function. It is of normal type $\left(t_{n} ; 1\right)$. If $\left\{t_{n}\right\}$ is an $A$ sequence and a given analytic function $F(x)$ is of normal type $\left\{t_{n} ; q\right\}$, then $F(x)$ is clearly an integral function. Hence the usefulness of $A$-sequences will be confined mainly, if not entirely, to the class of integral functions. It is obvious that (4) is necessary as well as sufficient to meet the requirement that every function of normal type $\left(t_{n} ; q\right)$ shall be an integral function.

We shall now develop necessary and sufficient conditions that $\left\{t_{n}\right\}$ shall be an $A$-sequence. We suppose condition (4) to be satisfied. Then $E(x)$ is an integral function. In order that $\left\{t_{n}\right\}$ shall be an $A$-sequence, it is necessary that for every positive number $a$ we have

$$
\limsup _{n=\infty}\left(t_{n} E^{(n)}(a) / n !\right)^{1 / n}=1 .
$$

This is obtained from (1) by taking $x_{0}=a, x_{1}=0$, and $F(x) \equiv E(x)$. But it is easily seen that the inferior limit of this limitand is not less than unity since for each value of $n$ the limitand itself is greater than unity. Consequently a necessary condition that a sequence $\left\{t_{n}\right\}$ of positive terms satisfying (4) shall be an $A$-sequence is that we shall have

$$
\lim _{n=\infty}\left(t_{n} E^{(n)}(a) / n !\right)^{1 / n}=1
$$

for every positive value of $a$.

Let us now suppose that $\left\{t_{n}\right\}$ is a sequence of positive terms satisfying conditions (4) and (6); and let $F(x)$ be a single-valued analytic function which is regular at the finite point $x_{0}$ for which

$$
\limsup _{n=\infty}\left|t_{n} F^{(n)}\left(x_{0}\right) / n !\right|^{1 / n}
$$

has the finite value $q=q\left(x_{0}\right)$. Then $F(x)$ is an integral function. Moreover, for every positive number $\epsilon$ there exists a positive number $M_{\epsilon}$ such that

$$
\left|F^{(\mu)}\left(x_{0}\right)\right|<M_{\epsilon}(q+\epsilon)^{\mu} \mu ! / t_{\mu}, \quad(\mu=0,1,2, \cdots) .
$$

Then from the power series expansion of $F^{(n)}(x)$ in powers of $x-x_{0}$ we have

$$
\begin{aligned}
\left|F^{(n)}(x)\right|<M_{\epsilon}(q+\epsilon)^{n} \sum_{\nu=0}^{\infty} & \frac{(n+\nu) !}{\nu !} \frac{1}{t_{n+\nu}}(q+\epsilon)^{\nu}\left|x-x_{0}\right|^{\nu} \\
& =M_{\epsilon}(q+\epsilon)^{n} E^{(n)}\left[(q+\epsilon)\left|x-x_{0}\right|\right] .
\end{aligned}
$$


Then from (6) with $a=(q+\epsilon)\left|x-x_{0}\right|$ and from the arbitrariness of the $\epsilon$ we see that

$$
\limsup _{n=\infty}\left|t_{n} F^{(n)}(x) / n !\right|^{1 / n}
$$

is not greater than $q$. If this superior limit were less than $q$ for some $x_{1}$, we could start from this $x_{1}$ and prove similarly that it is less than $q$ for $x=x_{0}$, contrary to hypothesis. Hence its value is $q$.

This enables us to conclude to the following theorem:

THEOREM II. A necessary and sufficient condition that a sequence $\left\{t_{n}\right\}$ of positive terms $t_{0}, t_{1}, t_{2}, \cdots$ shall be an $A$-sequence is that it shall satisfy condition (4) and the condition

$$
\lim _{n=\infty}\left(\sum_{\nu=0}^{\infty} \frac{t_{n}}{t_{\nu+n}} \frac{(\nu+n) !}{\nu ! n !} a^{\nu}\right)^{1 / n}=1
$$

for every positive value of $a$.

Equation (8) is merely another form of (6). An equivalent condition is set forth in the following corollary, which we now prove:

COROLlary 1. Condition (8) in the theorem may be replaced by the following equivalent condition:

$$
\lim _{n=\infty}\left(\sum_{\nu=0}^{\infty} \frac{t_{n}}{t_{v+n}} a^{\nu}\right)^{1 / n}=1 .
$$

Since the binomial theorem implies that

$$
(1+\delta)^{n+\nu} \geqq \frac{(\nu+n) !}{\nu ! n !} \delta^{\nu}
$$

for every positive $\delta$ and for non-negative integers $n$ and $\nu$, it follows that

$$
\frac{(\nu+n) !}{\nu ! n !} \leqq(1+\delta)^{n}\left(1+\frac{1}{\delta}\right)^{\nu}
$$

for every positive $\delta$. Hence we have

$$
\sum_{\nu=0}^{\infty} \frac{t_{n}}{t_{\nu+n}} \frac{(\nu+n) !}{\nu ! n !} a^{\nu} \leqq(1+\delta)^{n} \sum_{\nu=0}^{\infty} \frac{t_{n}}{t_{\nu+n}}\left(1+\frac{1}{\delta}\right)^{\nu} a^{\nu} .
$$

From this relation and from (9) with $a$ replaced by $a(1+1 / \delta)$ it follows that (9) implies (8). That (8) implies (9) follows readily from the fact that the limitand in (9) lies between 1 and the limitand in (8). Hence the corollary is established.

We shall now prove the following two corollaries of Theorem II: 
COROllary 2. Any one of the following is a necessary condition that the sequence $\left\{t_{n}\right\}$ be an A-sequence:
(a) $\lim _{n=\infty} \rho_{n}=\infty$,
(b) $\quad \lim _{n=\infty} \sup \lambda_{n}=\infty$,
(c) $\quad \lim _{n=\infty} \inf \left(\lambda_{n}\right)^{1 / n} \geqq 1$,
(d) $\lim _{n=\infty}\left(\lambda_{n+1} \lambda_{n+2} \cdots \lambda_{2 n}\right)^{1 / n}=\infty$.

COROLLARY 3. Either one of the following is a sufficient condition that the positive-termed sequence $\left\{t_{n}\right\}$ be an $A$-sequence:
(a) $\lim _{n=\infty} \lambda_{n}=\infty$,
(b) $\rho_{n+1} \geqq \rho_{n}, \lim _{n=\infty} \rho_{n}=\infty$.

In Corollary 2 (a) we have merely a repetition of Condition (4), and 2 (b) is an immediate consequence of 2 (a). Corollaries 2 (c) and 2 (d) follow immediately from a consideration of the second and the $(n+1)$ th terms in the series in (9). When the condition in Corollary 3 (a) is satisfied we have equation (4). When the same condition is satisfied, then for any $K$ whatever greater than $a$ we have

$$
1<1+\sum_{\nu=1}^{\infty} \frac{(n+\nu) !}{n ! \nu !} \frac{a^{\nu}}{\lambda_{n+1} \cdots \lambda_{n+\nu}}<\sum_{\nu=0}^{\infty} \frac{(n+\nu) !}{n ! \nu !} \frac{a^{\nu}}{K^{\nu}}=\left(1-\frac{a}{K}\right)^{-n-1}
$$

for all $n$ greater than a given value depending on $K$. Hence Condition (8) is satisfied. Thence from Theorem II we conclude to the truth of Corollary $3(\mathrm{a})$. This corollary in turn implies Corollary 3 (b).

By aid of Corollary 3 it is easy to show that the sequence $\left\{t_{n}\right\}$ is an $A$ sequence for each of the following definitions of $t_{n}$ :

(11) $t_{n}=(n !)^{t}$ or $t_{n}=e^{n+t}$ or $t_{n}=n^{t n}$ (except that $\left.t_{0}=1\right), \quad 0<t<\infty$;

(12) $t_{n}=e^{n(\log n)^{t}}, 0<t<\infty, n>0, t_{0}=1$;

(13) $t_{n}=(\log n)^{t n}$ or $t_{n}=n^{n\left[1-(\log n)^{-t}\right]}, 0<t<\infty, n \geqq 2, t_{0}=t_{1}=1$;

(14) $t_{n}=e^{i n l_{k}(n)}$ when $l_{k}(n)$ is real but otherwise $t_{n}=1$, where, in the last case, we have

$$
0<t<\infty, l_{1}(n)=\log n, l_{\mu}(n)=\log \left[l_{\mu-1}(n)\right], \mu>1 .
$$

Functions of normal type $(n ! ; q)$ are the functions of exponential type.* Functions of type $\left((n !)^{t} ; q\right)$, with $0<t<\infty$, and $0<q<\infty$, are the functions of order $1 / t$ and normal type $t q^{1 / t}$ in accordance with the classic terminology $\dagger$ of the theory of integral functions.

* See, for instance, R. D. Carmichael, Bulletin of the American Mathematical Socjety, vol. 40 (1934), pp. 241-261.

† See, for instance, A. Pringsheim, Mathematische Annalen, vol. 58 (1904), pp. 257-342. 
We shall now prove the following theorem:

THEOREM III. Let $\left\{t_{n}\right\}$ be an A-sequence. Let $\left\{t_{n}^{\prime}\right\}$, with $t_{0}^{\prime}=\lambda_{0}^{\prime}$, $t_{n}{ }^{\prime}=\lambda_{1}{ }^{\prime} \lambda_{2}^{\prime} \cdots \lambda_{n}{ }^{\prime}$ for $n=1,2,3, \cdots$, be any infinite sequence of positive numbers such that a positive constant $M$ exists so that $\lambda_{n}{ }^{\prime} \geqq M \lambda_{n}$ for $n=1,2, \cdots$. Then $\left\{t_{n}^{\prime}\right\}$ is an $A$-sequence.

Since $t_{n}$ satisfies Condition (4) it is evident that $t_{n}^{\prime}$ satisfies a like condition. For positive $n$ and positive $a$ we have

$$
1<\sum_{\nu=0}^{\infty} \frac{(n+\nu) !}{n ! \nu !} \frac{a^{\nu}}{\lambda_{n+1}^{\prime} \cdots \lambda_{n+\nu}^{\prime}} \leqq \sum_{\nu=0}^{\infty} \frac{(n+\nu) !}{n ! \nu !} \frac{(a / M)^{\nu}}{\lambda_{n+1} \cdots \lambda_{n+\nu}} .
$$

Hence Condition (8) on $t_{n}$ implies the corresponding condition on $t_{n}^{\prime}$. Thence it follows that Theorem III is implied by Theorem II.

4. Classification of integral functions. With respect to the general question of the existence of $A$-sequences suitable for classifying transcendental integral functions, we prove the following theorem:

THEOREM IV. For every transcendental integral function $F(x)$ there exists an associated $A$-sequence $\left\{t_{n}\right\}$ such that $F(x)$ is of type $\left(t_{n} ; 1\right)$ and the corresponding $\rho_{n}$ is monotonic non-decreasing.

We define the required sequence $\left\{t_{n}\right\}$ as follows. For positive $n$, let $t_{n}$ be a positive number such that $t_{n}^{-1 / n}$, or $1 / \rho_{n}$, is the greatest of the numbers

$$
\left|F^{(\mu)}(0) / \mu !\right|^{1 / \mu}, \quad(\mu=n, n+1, \cdots),
$$

such greatest numbers* obviously existing since $F(x)$ is a transcendental integral function. Put $\rho_{0}=\rho_{1}$. From Corollary $3(\mathrm{~b})$ to Theorem II it follows readily that such a sequence $\left\{t_{n}\right\}$ is an $A$-sequence, since $t_{n}^{-1 / n}$ is clearly a monotonic non-increasing function of $n$ approaching zero as $n$ becomes infinite, whence the corresponding $\rho_{n}$ is monotonic non-decreasing and becomes infinite with $n$. Thence it is easy to see that $F(x)$ is of type $\left(t_{n} ; 1\right)$ and that the theorem follows as stated.

The totality of $A$-sequences $\left\{t_{n}\right\}$ associated with the totality of transcendental integral functions in the manner indicated in the foregoing proof contains just those $A$-sequences for which $\rho_{n}$ is monotonic non-decreasing, as one sees from a consideration of the particular function

$$
F(x)=1+\rho_{1}^{-1} x+\rho_{2}^{-2} x^{2}+\cdots .
$$

5. Bounds on functions of normal type $\left(t_{n} ; q\right)$ for $A$-sequences $\left\{t_{n}\right\}$. Equa-

* Such sequences $\left\{1 / \rho_{n}\right\}$ have been considered by G. Faber, Mathematische Annalen, vol. 70 (1911), pp. 48-68; see p. 51 . 
tion (7) affords an upper bound to $\left|F^{(n)}(x)\right|$ in terms of the $n$th derivative of $E(x)$ when $F(x)$ is a function of normal type $\left(t_{n} ; q\right)$ and $\left\{t_{n}\right\}$ is an $A$-sequence. It is sometimes useful to have such an upper bound in terms of $E(x)$ itself rather than in terms of its derivatives. In this connection we prove the following theorem:

THEOREM V. Let $\left\{t_{n}\right\}$ be an A-sequence such that the corresponding $\rho_{n}$ is monotonic non-decreasing. Then if $F(x)$ is of normal type $\left(t_{n} ; q\right)$, we have for every positive $\delta$ and every positive $\epsilon$ an $M_{\epsilon}\left(x_{0}\right)$ depending on $\epsilon$ and $x_{0}$ alone such that

$$
\left|t_{n} F^{(n)}(x) / n !\right|<M_{\epsilon}\left(x_{0}\right)(q+\epsilon)^{n}(1+1 / \delta)^{n} E\left[(q+\epsilon)(1+\delta)\left|x-x_{0}\right|\right]
$$

for every finite $x$ and $x_{0}$.

From (7) and (10) we have $\left|t_{n} F^{(n)}(x) / n !\right|<M_{\epsilon}\left(x_{0}\right)(q+\epsilon)^{n}(1+1 / \delta)^{n} \sum_{\nu=0}^{\infty} \frac{t_{n}}{t_{n+\nu}} \cdot(q+\epsilon)^{\nu}(1+\delta)^{\nu}\left|x-x_{0}\right|^{\nu}$.

But since $\rho_{n}$ is now monotonic we have for $n \nu>0$ the relations

$$
t_{n} t_{\nu}=\rho_{n}^{n} \rho_{\nu}^{\nu} \leqq \rho_{n+\nu}^{n} \rho_{n+\nu}^{\nu}=t_{n+\nu} \text {, whence } t_{n} / t_{n+\nu} \leqq 1 / t_{\nu} \text {. }
$$

From these relations and the definition of $E(x)$ we have (15), and the theorem is proved.

6. Type of the derived function. We now prove the following theorem:

THEOREM VI. If $F(x)$ is of type $\left(t_{n} ; q\right)$, for a given $A$-sequence $\left\{t_{n}\right\}$, then $F^{\prime}(x)$ is of type $\left(t_{n} ; q_{1}\right)$ where $q_{1} \leqq q$. When $q$ is finite, a necessary and sufficient condition that $q_{1}=q$ in all cases is that $\lim _{n=\infty}\left(\lambda_{n}\right)^{1 / n}=1$.

We have

$$
\begin{aligned}
\limsup _{n=\infty}\left|t_{n} F^{(n+1)}\left(x_{0}\right) / n !\right|^{1 / n}= & \limsup _{n=\infty}\left|t_{n} F^{(n+1)}\left(x_{0}\right) / n !\right|^{1 /(n+1)} \\
\leqq & \lim _{n=\infty}\left|t_{n+1} F^{(n+1)}\left(x_{0}\right) /(n+1) !\right|^{1 /(n+1)} \\
& \cdot \limsup _{n=\infty}\left(1 / \lambda_{n+1}\right)^{1 /(n+1)} .
\end{aligned}
$$

Now apply Corollary 2(c) following Theorem II. The stated theorem follows readily.

7. Representation of functions of normal type $\left(t_{n} ; q\right)$. We prove the following theorem:

THEOREM VII. $A$ necessary and sufficient condition that a function $F(x)$ be of normal type $\left(t_{n} ; q\right)$, for a given $A$-sequence $\left\{t_{n}\right\}$, is that it be expressible in the form 


$$
F(x)=\frac{1}{2 \pi i} \int_{C} E(x z) a(z) d z,
$$

where $a(z)$ is a function which is analytic in the ring $q<|z| \leqq f \leqq \infty$ and has a singularity on the circle $|z|=q$, and where $C$ is a circle of radius $q+\epsilon$, $0<\epsilon<f-q$, about the point 0 as a center. Here $E(x)$ is the function defined in (3).

An easily verified representation of $F(x)$ in the form indicated is the following:

$$
F(x)=\frac{1}{2 \pi i} \int_{C} E(x z)\left(\sum_{n=0}^{\infty} \frac{t_{n} F^{(n)}(0)}{n ! z^{n+1}}\right) d z .
$$

Conversely, if $F(x)$ has the representation (16), then we may write

$$
a(z)=\sum_{\nu=0}^{\infty} \frac{a_{\nu}}{z^{\nu+1}}+\sum_{\nu=0}^{\infty} b_{\nu} z^{\nu}, \quad q<|z| \leqq f,
$$

where $\lim \sup _{\nu=\infty}\left|a_{\nu}\right|^{1 / \nu}=q$. Then from (16) we have

$$
F(x)=\sum_{\nu=0}^{\infty} \frac{a_{\nu} x^{\nu}}{t_{\nu}}
$$

whence it follows readily that $F(x)$ is of normal type $\left(t_{n} ; q\right)$.

8. On $B$-sequences. Independently of the notion of $A$-sequence, we shall now define a class of sequences, which we call $B$-sequences, and we shall then prove that every $B$-sequence is an $A$-sequence.

Let $\left\{t_{n}\right\}$ be an infinite sequence of positive numbers $t_{0}, t_{1}, t_{2}, \ldots$ verifying equation (4). Define $E(x)$ as in (3). If $F(x)$ is a single-valued analytic function which is regular at $x=x_{0}$ and if we have

$$
\limsup _{n=\infty}\left|t_{n} F^{(n)}\left(x_{0}\right) / n !\right|^{1 / n}=q=q\left(x_{0}\right), \quad(0 \leqq q<\infty),
$$

whence $F(x)$ is an integral function, then for every positive number $\epsilon$ there exists a constant $M_{\epsilon}\left(x_{0}\right)$, depending on $\epsilon$ and $x_{0}$, such that

$$
|F(x)|<M_{\epsilon}\left(x_{0}\right) E\left[(q+\epsilon)\left|x-x_{0}\right|\right]
$$

for every finite $x$, as we may see through a proof similar to that by which equation (7) was derived.

Let us suppose conversely that the sequence $\left\{t_{n}\right\}$ verifies (4) and is such that every integral function $F(x)$ having the property associated with (18) has also the property that

$$
\limsup _{n=\infty}\left|t_{n} F^{(n)}\left(x_{0}\right) / n !\right|^{1 / n} \leqq q .
$$


Then, and only then, we shall say that $\left\{t_{n}\right\}$ is a $B$-sequence.

THEOREM VIII. Every B-sequence is an A-sequence.

Let $\left\{t_{n}\right\}$ be a $B$-sequence. Define $E(x)$ as in equation (3). We have

$$
\begin{aligned}
|E(x)| \leqq E(|x|) & =\sum_{\nu=0}^{\infty} \frac{\left|x-x_{0}\right|^{\nu}}{t_{\nu}}\left|1+\frac{x_{0}}{x-x_{0}}\right|^{\nu} \\
& \leqq M_{\epsilon}\left(x_{0}\right) E\left[(1+\epsilon)\left|x-x_{0}\right|\right] .
\end{aligned}
$$

Then, since $\left\{t_{n}\right\}$ is a $B$-sequence, we have

$$
\limsup _{n=\infty}\left|t_{n} E^{(n)}\left(x_{0}\right) / n !\right|^{1 / n} \leqq 1 .
$$

Letting $x_{0}$ run over all positive values $a$ we evidently have the condition associated with (8), since the limitand is never less than 1 . Since $t_{n}$ verifies (4), it follows from Theorem II that $\left\{t_{n}\right\}$ is an $A$-sequence, as was to be proved.

THEOREM IX. A sufficient condition that a sequence $\left\{t_{n}\right\}$ of positive numbers $t_{0}, t_{1}, t_{2}, \cdots$ satisfying (4) shall be a $B$-sequence is that positive numbers $r_{n}$ shall exist such that we have

$$
\lim _{n=\infty}\left\{\sum_{\nu=0}^{\infty} \frac{r_{n}{ }^{\nu}}{r_{n}^{n}} \frac{t_{n}}{t_{\nu}}\right\}^{1 / n}=1
$$

Let $F(x)$ be a single-valued analytic function verifying a relation of the form (18) where $\left\{t_{n}\right\}$ is a sequence verifying (4). Then $F(x)$ is an integral function, and we may write

$$
\frac{F^{(n)}\left(x_{0}\right)}{n !}=\frac{1}{2 \pi i} \int_{\Gamma_{n}} \frac{F(z)}{\left(z-x_{0}\right)^{n+1}} d z,
$$

where $\Gamma_{n}$ is a circle of radius $r_{n} /(q+\epsilon)$ about the point $x_{0}$ as a center. From (18) and the last relation we have

$$
\left|t_{n} F^{(n)}\left(x_{0}\right) / n !\right| \leqq M_{\epsilon}\left(x_{0}\right)(q+\epsilon)^{n} t_{n} r_{n}{ }^{-n} E\left(r_{n}\right)=M_{\epsilon}\left(x_{0}\right)(q+\epsilon)^{n} \sum_{\nu=0}^{\infty} \frac{r_{n}^{\nu}}{r_{n}^{n}} \frac{t_{n}}{t_{\nu}} .
$$

If a sequence $r_{n}$ exists for which (20) is satisfied, then we see that (19) is satisfied and hence that $\left\{t_{n}\right\}$ is a $B$-sequence.

TheOREM X. A sufficient condition that the sequence $\left\{t_{n}\right\}$ of positive numbers $t_{0}, t_{1}, t_{2}, \cdots$ be a $B$-sequence is that the corresponding sequence $\left\{\lambda_{n}\right\}$ be monotonic non-decreasing and have infinity as a limit.

It is clear that condition (4) is satisfied. It is easy to verify that the hypotheses imply the relations 


$$
\lambda_{n}{ }^{\nu-n} t_{n} / t_{\nu} \leqq 1, \quad(\nu, n=1,2, \cdots) .
$$

Hence, if we take $r_{n}=(1-1 / n) \lambda_{n}$ for $n>1$, we have

$$
\begin{aligned}
1 \leqq \sum_{\nu=0}^{\infty} \frac{r_{n}^{\nu}}{r_{n}^{n}} \frac{t_{n}}{t_{\nu}} \leqq & \sum_{\nu=0}^{\infty}\left(1-\frac{1}{n}\right)^{\nu-n}+\left(\frac{1}{t_{0}}-1\right)\left(1-\frac{1}{n}\right)^{-n} \\
& =\left(n+\frac{1}{t_{0}}-1\right)\left(1-\frac{1}{n}\right)^{-n}, \quad n>1 .
\end{aligned}
$$

Then equation (20) follows. Then Theorem $\mathrm{X}$ follows from Theorem IX.

TheOREM XI. A necessary condition that the sequence $\left\{t_{n}\right\}$ of positive numbers $t_{0}, t_{1}, t_{2}, \cdots$ be a $B$-sequence is that we have

$$
\liminf _{n=\infty}\left(\lambda_{n+1} / \lambda_{n}\right)^{1 / n} \geqq 1 .
$$

We shall prove this by showing that a contradiction arises if we assume that a $B$-sequence $\left\{t_{n}\right\}$ exists for which we have

$$
\liminf _{n=\infty}\left(\lambda_{n+1} / \lambda_{n}\right)^{1 / n}=\theta<1 .
$$

Let $\theta_{1}$ and $\theta_{2}$ be positive numbers such that $\theta<\theta_{1}{ }^{2}<\theta_{1}<\theta_{2}<1$. Then we have

$$
\lambda_{n+1}<\theta_{1}{ }^{2 n} \lambda_{n}<\theta_{1}^{n} \lambda_{n}
$$

for an infinite increasing sequence of positive values $n_{0}, n_{1}, n_{2}, \cdots$ of $n$. Then for such $n$ we have

$$
\begin{aligned}
& \frac{|x|^{n}}{t_{n} \theta_{2}{ }^{n}}=\frac{|x|^{n-1}}{t_{n-1}}\left(\frac{\theta_{1}}{\theta_{2}}\right)^{n} \frac{|x|}{\theta_{1}{ }^{n} \lambda_{n}} \leqq \frac{|x|^{n-1}}{t_{n-1}}\left(\frac{\theta_{1}}{\theta_{2}}\right)^{n} \leqq E(|x|)\left(\frac{\theta_{1}}{\theta_{2}}\right)^{n} \\
& \quad \text { if }|x| \leqq \theta_{1}^{n} \lambda_{n}, \\
& \frac{|x|^{n}}{t_{n} \theta_{2}{ }^{n}}=\frac{|x|^{n+1}}{t_{n+1}}\left(\frac{\theta_{1}}{\theta_{2}}\right)^{n} \frac{\theta_{1}^{-n} \lambda_{n+1}}{|x|} \leqq \frac{|x|^{n+1}}{t_{n-1}}\left(\frac{\theta_{1}}{\theta_{2}}\right)^{n} \leqq E(|x|)\left(\frac{\theta_{1}}{\theta_{2}}\right)^{n} \\
& \text { if }|x| \geqq \theta_{1}^{-n} \lambda_{n+1} .
\end{aligned}
$$

Since $\theta_{1}^{-n} \lambda_{n+1}<\theta_{1}^{n} \lambda_{n}$ for such $n$ the two ranges of $x$ cover all finite values of $x$. Hence, for the function

$$
F(x)=\sum_{\nu=0}^{\infty} \frac{x^{n_{\nu}}}{t_{n_{\nu}} \theta^{{ }^{n}{ }^{\nu}}}
$$

we have the relation

$$
|F(x)| \leqq F(|x|) \leqq \frac{\theta_{2}}{\theta_{2}-\theta_{1}} E(|x|) .
$$


But

$$
\limsup _{n=\infty}\left|t_{n} F^{(n)}(0) / n !\right|^{1 / n}=\frac{1}{\theta_{2}}>1 .
$$

The last two relations contradict the hypothesis that $\left\{t_{n}\right\}$ is a $B$-sequence. Hence the theorem follows as stated.

The foregoing theorem will enable us to prove the following:

THEOREM XII. Not every A-sequence is a B-sequence.

Let $\left\{t_{n}\right\}$ denote the sequence for which we have

$$
\rho_{0}=1, \quad \rho_{n}=2^{\nu} \text { for } 3 \cdot 2^{\nu-2}<n \leqq 3 \cdot 2^{\nu-1}, \quad(\nu=0,1,2, \cdots) .
$$

Since $\rho_{n}$ is monotonic non-decreasing and has the limit infinity it follows from Corollary 3 to Theorem II that $\left\{t_{n}\right\}$ is an $A$-sequence. But from Theorem XI it follows that it is not a $B$-sequence, since we now have

$$
\liminf _{n=\infty}\left(\lambda_{n+1} / \lambda_{n}\right)^{1 / n} \leqq \frac{1}{2}
$$

as one sees by considering the values $3 \cdot 2^{n-2}+1$ of $n$. Hence the theorem is established.

The sequences $\left\{t_{n}\right\}$ defined in equations (11) to (14) are $B$-sequences, as one shows by aid of Theorem $\mathrm{X}$ and the fact that $\lambda_{n} \geqq \rho_{n}$ when $\rho_{n}$ is monotonic non-decreasing, owing to the relation $\lambda_{n}=\rho_{n}{ }^{n}\left(\rho_{n-1}\right)^{-n+1}$ which holds in all cases for positive $n$.

9. Necessary and sufficient conditions for normal type $\left(t_{n} ; q\right)$. We have the following theorem:

THEOREM XIII. Let $\left\{t_{n}\right\}$ be a B-sequence. Then a necessary and sufficient condition that a single-valued analytic function $F(x)$ shall be of normal type $\left(t_{n} ; q\right)$ is that (a) numbers $p$ shall exist for which it is true that for every positive number $\epsilon$ there exists a constant $M$, depending on $\epsilon$ and $p$ but independent of $x$, such that for all finite values of $x$ we have

$$
\cdot|F(x)|<M E[(p+\epsilon)|x|]
$$

and (b) that $q$ shall be the least of such numbers $p$.

Moreover, when $F(x)$ is of normal type $\left(t_{n} ; q\right)$ we have

$$
\left|F^{(n)}(x)\right|<M(q+\epsilon)^{n} E^{(n)}[(q+\epsilon)|x|], \quad(n=0,1,2, \cdots),
$$

where $M$ is independent of $x$ and $n$.

The proposition in the first paragraph of the theorem is an immediate consequence of the earlier part of $\S 8$; that in the second paragraph follows from ( 7$)$ and the fact that every $B$-sequence is an $A$-sequence. 
Pringsheim (loc. cit., pp. 273, 280) has observed that the sequence $\left\{(n !)^{1 / t}\right\}, 0<t<\infty$, possesses the properties characterizing both our $A$-sequences and our $B$-sequences. Results obtained by him imply the following proposition:

A necessary and sufficient condition that a single-valued analytic function $F(x)$ shall be of normal type $\left\{(n !)^{1 / t} ; q\right\}, 0<t<\infty, 0<q<\infty$, that is to say, of the classic order $t$ and normal type $q^{t} / t$, is that (a) numbers $p$ shall exist for which it is true that for every positive number $\epsilon$ there exists a quantity $M$, depending on $\epsilon$ and $p$ but independent of $x$, such that for all finite values of $x$ we have

$$
|F(x)|<M e^{(p+\epsilon)^{t}|x|^{t} / t}
$$

and (b) that $q$ shall be the least of such numbers $p$.

From this result and our Theorem $\mathrm{V}$ we have the following theorem:

TheOREM XIV. If $F(x)$ is of normal type $\left\{(n !)^{1 / t} ; q\right\}, 0<t<\infty$, then, for every positive $\eta, \epsilon, \delta$, there exists a constant $M_{\eta \in \delta}$, independent of $x$ and $n$, such that for all $n$ and for all finite $x$ we have the inequality:

$$
\left|(n !)^{1 / t} F^{(n)}(x) / n !\right|<M_{\eta \epsilon \delta}(q+\epsilon)^{n}\left(1+\frac{1}{\delta}\right)^{n} e^{(q+\epsilon)^{t}(1+\delta)^{t}(1+\eta)^{t}|x|^{t} / t} .
$$

UNIVERSITY OF ILLINOIS,

URBANA, ILL. 